\title{
Intelligent Fault Diagnosis of Military Power Based on BP Neural Network
}

\author{
Rui ZHANG ${ }^{a,}$, , Bo FAN ${ }^{b}$, Xinyu LUAN ${ }^{a}$ \\ ${ }^{1}$ Air force Engineering University, College of Air and Missile Defense, Xi'an, 710051,China \\ a18681949708@163.com, ${ }^{\text {a } f a n b o 2000 @ 163 . c o m, ~}{ }^{*}$ corresponding author
}

Keywords: neural network; three phase DC/AC inverter; fault diagnosis

\begin{abstract}
The reliable operation of power equipment related to the performance of surface-to-air missile weapon systems, accurate fault diagnosis of power system is very important. In order to make an accurate diagnosis for power supply equipment of the surface-to-air missile weapon systems, this paper introduces the BP neural network and the related knowledge of some type missile static variable power supply. The fault model of three phase DC/AC inverter is established, and several common faults are analyzed briefly. BP neural model is applied to the fault diagnosis of a certain type of ground to air missile static variable power, the neural network's ability to classify the pattern is good, solve the previous static inverter fault diagnosis problems of surface to air missile troops. The simulation results show that the method can diagnose the fault of power equipment accurately, and the accuracy and practicability of the method are verified.
\end{abstract}

\section{Introduction}

Power supply equipment is one of the important parts of ground to air missile weapon system. The power supply of the system is all from power supply equipment. The quality of the power supply provided by the power supply equipment is related to the performance of the weapon system. The electric energy of ground to air missile mainly comes from mobile power station. In order to reduce the operation cost, a mobile power station is equipped with a frequency solid power supply. When input Commercial power, the diesel generator set can't be started, and the power supply is supplied by frequency solid power supply. How to ensure the normal operation and reliable power supply of frequency solid power supply in daily training is an urgent to be solved.

The core component of frequency solid power supply is three-phase DC/AC inverter, which uses PWM control. Practical verification showed that the inverter was the key and difficult point of frequency solid power supply fault diagnosis. At present, the research on the reliability of the ground to air missile weapon system diesel generator sets is relatively large, because use of the frequency solid power supply is not long, the research level on this area is still relatively low. High tech weapons power system urgently requires modern technical means to help power equipment workers quickly analyze and deal with various practical problems and ensure the normal and safe operation of power equipment.

Artificial neural network (ANN) has many advantages, such as association, memory, storage and learning, and it is widely used in the field of fault diagnosis. In this paper, the fault diagnosis of three-phase DC/AC inverter is carried out by using BP neural network model. Through spectral analysis method to preprocess the sample data, analyzed several common faults of three-phase DC/AC inverter and got the correct diagnosis, provides new ideals and new methods for fault diagnosis of frequency solid power supply of primary surface to air missile system.

\section{BP Network Classifier}

BP neural network is one of the most important components of artificial neural networks. Most of the artificial neural network models adopt the BP network model or its charge form. The BP neural network model has been widely used in function approximation, pattern recognition, data compression and so on [1-2].

BP Neurons and Models. The difference between BP neurons and other neurons is that BP 
neurons generally used nonlinear function as transfer function, such as the most commonly used logsig and tansig function, but a few of output layers use linear function (purelin) as transfer function. As shown in Figure 1.

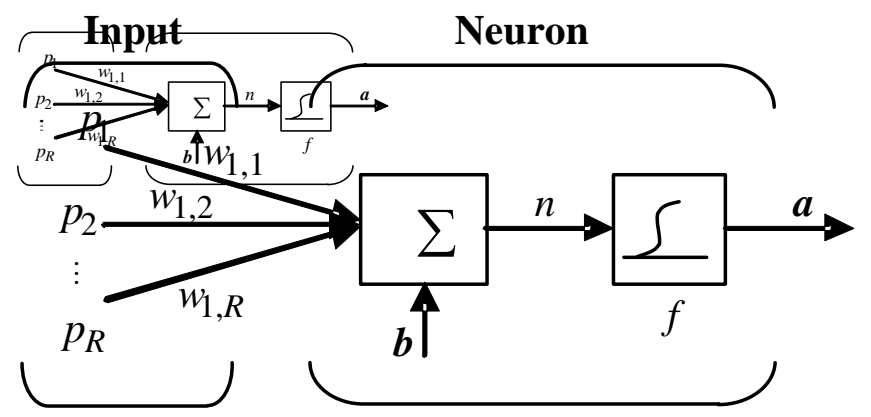

Figure 1 BP Neurons

BP neural network usually adopts the form of multilayer neural network, and the double-layer network constructed by BP neural network is shown in Figure 2. The information in the BP neural network is transmitted from the input layer, and the error is transmitted from the output layer to the input layer. It can be seen that it is a multi-layer feed-forward neural work model [3-4].

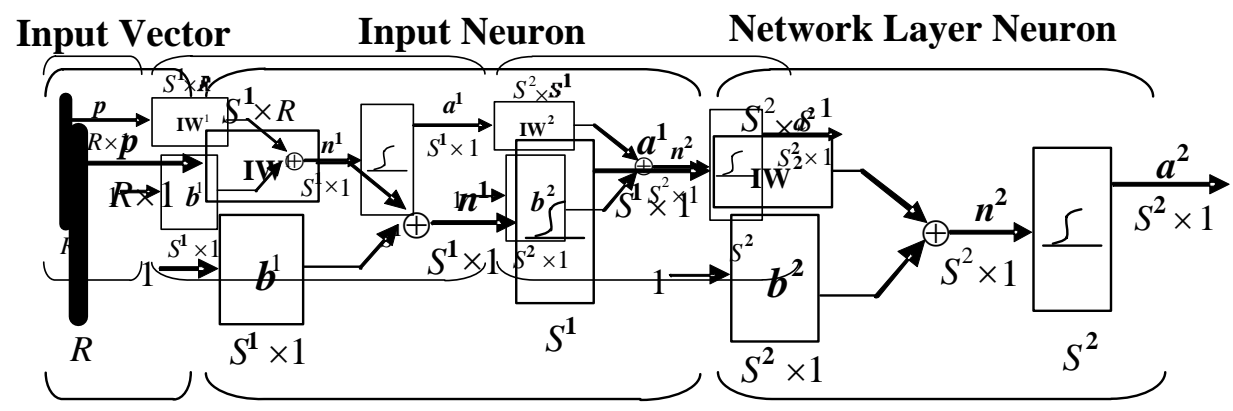

Figure 2 Double-layer BP Neural Network

If the output layer of the multilayer BP neural network uses the nonlinear $\mathrm{S}$ type transfer function (such as logsig), its output value will be limited to a small area, but the linear transfer function can be arbitrarily valued.

Learning of BP Networks. The learning process of BP network can be divided into two processes, the forward transfer of signals and the reverse transmission of errors. When forward transfer, the sample signals are processed by the input layer, the hidden layer and the output layer in order to get the output signal, the actual output signal is compared with the desired output signal, and if the error of the two exceeds the set point, the reverse phase of the steering error signal is transmitted. Error reverse transfer is the reverse of the error between the actual output and the expected output, from the output layer to the input layer through the hidden layer, and transfer the error to each part of each layer. At this point, the error signal can be considered as the reference data for the weight modification. The process of adjusting the weights of each layer by signal forward transfer and error reverse transmission is called the learning process of the network. This learning process in BP networks will end when the output error reaches an acceptable range or reaches the set number of learning steps [5].

\section{Fault Model of Three-Phase DC/AC Inverter}

A static frequency conversion power supply is an alternative power source for diesel generators. When there have Commercial power, the diesel generator set can not be started, and the static frequency conversion power supply is used to provide training, detection and test power supply for the power supply equipment. In this way, the operation cost of power supply car and maintenance cost of power unit can be reduced, and the service life of power supply car will be improved.

The power of this static frequency conversion power supply is large, which consists of a host computer and a slave computer. The core of the static frequency conversion power supply is a DC/AC inverter. 
From the DC power source to the load terminal, the three-phase DC/AC inverter may encounter 9 fault types as shown in figure 3.

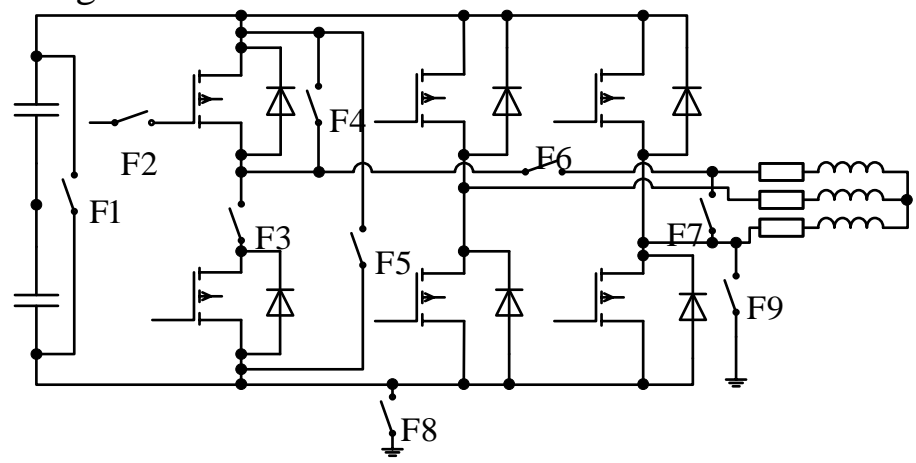

Figure 3 Fault model of three-phase DC/AC inverter

The fault type at F1 in figure 3 is the short circuit of the DC bus capacitor. Statistical data show that the faults of aluminum electrolytic capacitors account for $83 \%$ of the short-circuit faults and $17 \%$ of the open circuit faults. Since the DC bus capacitor usually uses aluminum electrolytic capacitor, the fault type is usually short circuit fault.

The fault type at F2 is the power switch without driving signal, which is usually due to the fault of the control circuit. Statistical data show that the fault is mainly caused by the optocoupler failure of the drive, but also caused by the gate fault of the power switch itself. The fault mode causes an open circuit failure of the power switch in the inverter.

The fault type at F3 is the half bridge open fault of a phase bridge arm, the reasons for this are: (1) An open circuit fault occurs on a drive on the bridge arm path. (2) The fuse on the bridge arm is blown off. (3) Separation of connecting points on bridge arm.

The fault type at F4 is power switch short circuit fault. Most of the faults are caused by the reverse breakdown of the power switch, and may also be due to the buffer switch circuit is connected in parallel at both ends of the insulation problems or control circuit fault short circuit fault, the bridge arm and lead driver of the switch signal can't be changed, so that the switch has been conducted and it can't be turned off.

The fault type at F5 is one phase bridge arm straight through, Most of the faults are considered as cascading failures due to F4 failures. When a power switch is short circuited, but another power switch on the same bridge arm did not turn off, so that the bridge arm short-circuit occurs, motor speed control system is most vulnerable to the threat of failure.

The fault type at F6 is one phase in the inverter open, There may be some reasons: (1) Separation of junction points in a phase. (2) The filter inductance connected in series between the inverter bridge arm and the load is open. This kind of fault is equivalent to the whole upper and lower arm of a bridge open.

The fault type at F7 is the two phase output short-circuit of the inverter, the reasons of the fault are: (1) The filter capacitor connected to the two phase is short circuited. (2) The insulation of the connecting cable is out of action.

The fault type at F8 is DC bus grounding. The fault type at F8 is inverter connected to the ground. The insulation failure of cables is the main factor of the two faults.

The actual operation results show that most of the faults in three-phase DC/AC inverter are caused by the damage of power switch devices, among which the open circuit and short circuit of power switch device are the most common. So in this paper, the main discussion is power switch no drive signal (F2) and power switch short circuit (F4) [6].

\section{Three-Phase DC/AC Inverter Circuit Fault Diagnosis Simulation}

As mentioned above, the failure of the actual system operation is analyzed. At the same time, in order to simplify the analysis process, the main circuit IGBT fault of the inverter is divided into 22 cases: single pipe fault and two pipe faults at the same time [7]. To enable neural networks to identify all fault modes, these fault patterns are encoded with six bit encoding. Fault patterns can be 
divided into five broad categories:

The first category (001): The power semiconductor IGBT has no trouble, the normal operation (000).

The second category (010): one IGBT fault is divided into six categories: (1) V1 fault (001). (2) V2 fault (010). (3) V3 fault (011). (4) V4 fault (100). (5) V5 fault (101). (6) V6 fault (110).

The third category (011): two different IGBT fault the same branch bridge arm is divided into three categories: (1) V1 and V4 fault (001). (2) V3 and V6 fault (010). (3) V5and V2 fault (011).

The fourth category (100): two IGBT fault of different branches and different bridge arms is divided into six categories: (1) V1 and V6 fault (001). (2) V1 and V2 fault (010). (3) V3 and V2 fault (011). (4) V3 and V4 fault (100). (5) V5 and V4 fault (101). (6) V5 and V6 fault (110).

The fifth category (101): two IGBT of different branches and same bridge arm fault is divided into six categories: (1) V1 and V3 fault (001). (2) V1and V5 fault (010). (3) V3 and V5 fault (011). (4) V4 and V6 fault (100). (5) V4 and V2 fault (101). (6) V6 and V2 fault (110).

In the MATLAB/SIMULINK environment, the model is set up, and the waveform of the output line voltage $V_{U V}$ of the inverter is obtained by simulating different fault conditions. Figure 4 shows the waveform of the inverter normal operation, a IGBT circuit fault and two IGBT (the same branch) at the same time open circuit fault occurred when the inverter output line voltage $V_{U V}$.

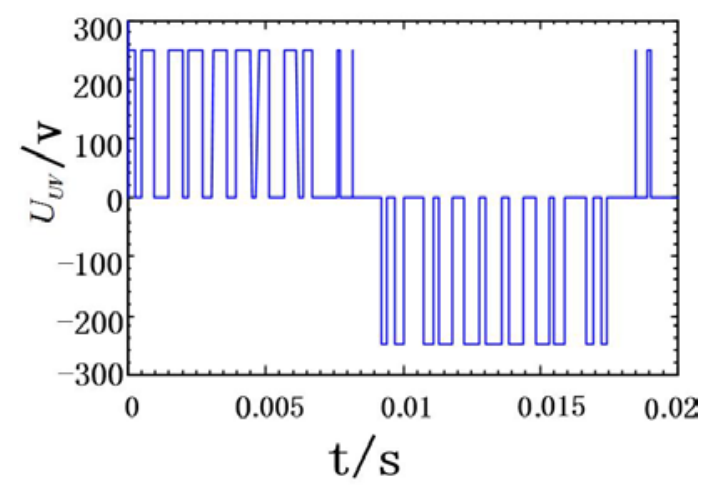

(a) Normal operation $V_{U V}$ waveform

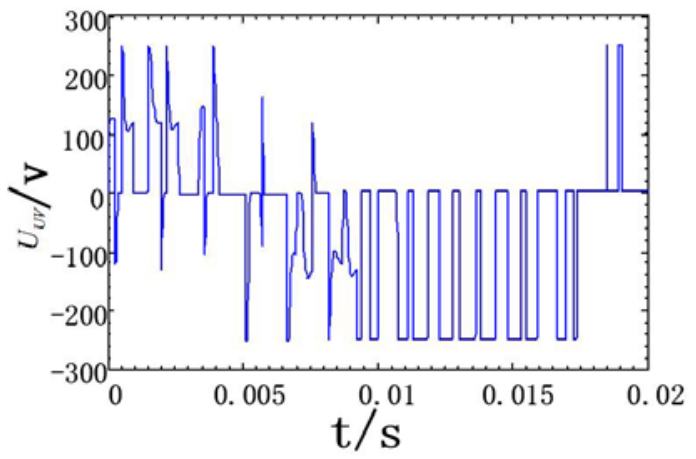

(b) One IGBT fault $V_{U V}$ waveform

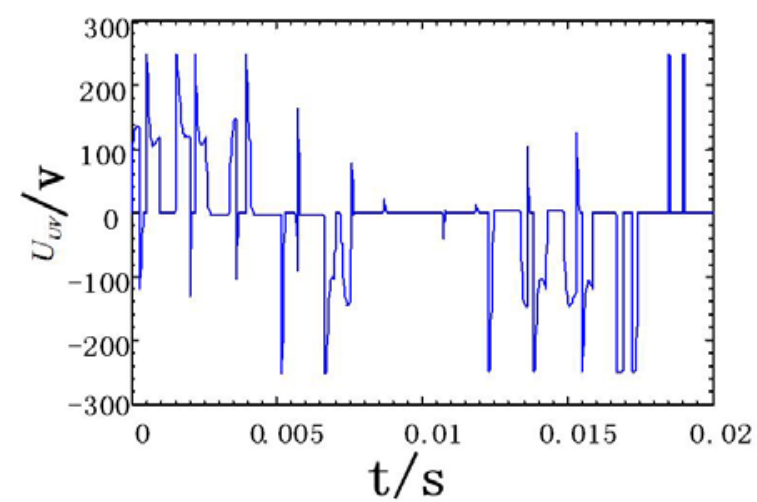

(c) two IGBT faults at the same time $V_{U V}$ waveform

Fig. $4 V_{U V}$ simulation waveforms of normal operation and fault 
In the time domain analysis of $V_{U V}$ waveform to obtain fault signal, the computation will be very large. Therefore, the $V_{U V}$ waveform signals in time domain are usually transformed into frequency domain by means of Fourier transform, and obtain the DC component, fundamental amplitude, phase, 2 harmonic phase 4 characteristic signal data. In frequency domain, these features of $V_{U V}$ signal essentially contain all the information used for fault identification. These signals are input to the input as the input signal of the neural network. In order to reduce the amount of calculation, the characteristic signal data is normalized, that is the sample data is expressed by per-unit values. The input voltage is selected as $250 \mathrm{~V}$, every kind of fault characteristic signal and the corresponding fault code under different loads constitute training samples and test samples.

In this paper, the MATLAB toolbox is used to study the fault diagnosis of a three phase DC/AC inverter for a ground to air missile static power supply. The neural network training error curve is shown in figure 5. The training samples and the sample data are normalized, and the Fourier transform is performed. Table 1 show the fault as the text sample when the load power is $100 \mathrm{~kW}$, and the actual output is compared with the expected output after the training of the neural network. The $Y_{i} \geq 0.5$ in table is 1 , and $Y_{i}<0.5$ is 0 . The fault diagnosis results can be obtained by querying the fault codes. The simulation results showed that the fault diagnosis was more accurate and the fault category was verified, which verified the validity and accuracy of the method.

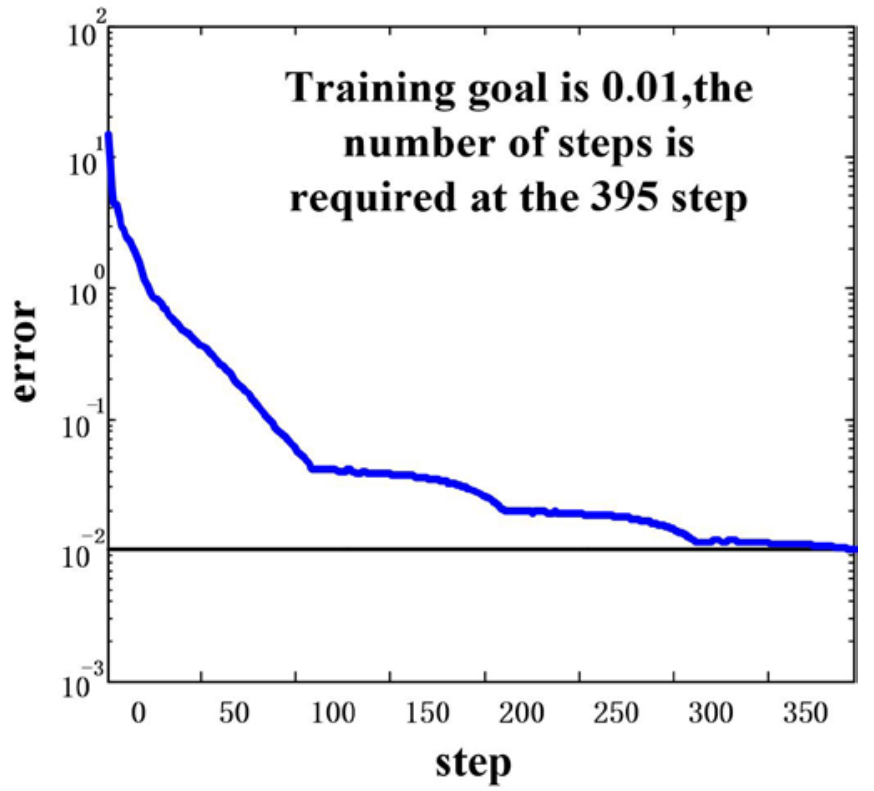

Fig. 5 training error curve

Table 1 test results of neural network when load 100KW

\begin{tabular}{cccccccc}
\hline Experimental serial number & \multicolumn{1}{c}{ Actual output } & Desired output \\
\hline 1 & -0.050 & 0.110 & 1.124 & 0.452 & 0.471 & -1.078 & 001000 \\
\hline 2 & -0.052 & 0.986 & -0.042 & -0.019 & 0.158 & 0.756 & 010001 \\
\hline 3 & 0.042 & 1.027 & 1.008 & 0.056 & -0.024 & 0.923 & 011001 \\
\hline 4 & 1.106 & -0.078 & -0.085 & -0.950 & -0.461 & 2.863 & 100001 \\
\hline 5 & 0.055 & 0.990 & 0.867 & 0.321 & 0.759 & 0.148 & 011010 \\
\hline
\end{tabular}

\section{Conclusion}

In this paper, the fault model of a static frequency conversion power supply inverter is established, and several common faults are studied. In this paper, the fault characteristic vectors are obtained by frequency spectrum analysis method, and the BP neural network model is used to diagnose the faults of the inverter. The simulation results show that this method is practical and effective. 


\section{References}

[1] WU Xiao-hua, SHI Zhong-ke, ZHANG Xiao-bin. Research and design of on line fault diagnosis for three-phase inverter based on DSP [J]. Power electronic technology, 2004,38(2):37-39.

[2] BI Tian-shu, NI Yi-xin, WU Fu-li, ZHOU Cui-cui. Fault diagnosis system based on hybrid neural network and genetic algorithm[J]. Modern power, 2005,22(1):31-36.

[3] Li Maojun, Tong Tiaosheng, A further result on the schema theorem of partheno-genetic algorithm, Control Theory and Application,2001,18(3):465 468

[4] Niska H, Hiltunen T, Karppinen A, Ruuskanen J, Kolehmainen M, Evolving the neural network model for forecasting air pollution time series, Engineering Applications of Artificial Intelligence,March,2004,17(2):159 167

[5] ZHOU Hui-ren, ZHEN Pi-e. Time series prediction based on hierarchical genetic algorithm and BP network[J]. Journal of systems simulation, 2007, 19(21):5055-5058.

[6] CHEN Hui-qin. Theory and application of genetic algorithm based on artificial neural network[D]. Wu Han, Wuhan University of Technology, 2003.

[7] LIANG Hong, WANG Qiu-yan. Fault diagnosis of three phase SPWM inverter circuit based on rough set and neural network [J]. Journal of Liaoning Institute of Technology, 2005,25(6):351-353. 